\title{
Influencia sobre la hospitalización de una consulta monográfica para pacientes con insuficiencia cardiaca dirigida por internistas. Estudio de cohortes
}

\author{
Impact of a monographic clinic managed by internist for patients whit chronic \\ heart failure on hospital admission rates. A cohort study
}

\author{
Jose Manuel Cerqueiro González¹, C. Abeledo Vázquez², S. de la Fuente Sánchez ${ }^{3}$, Emilio Casariego Vales ${ }^{1}$ \\ ${ }^{1}$ Servicio de Medicina Interna. Hospital Universitario Lucus Augusti. SERGAS. Lugo \\ ${ }^{2}$ Personal de enfermería. Medicina Interna. Hospital Universitario Lucus Augusti. SERGAS. Lugo \\ ${ }^{3}$ Médico Residente en Medicina Familiar y Comunitaria. Hospital Universitario Lucus Augusti. SERGAS. Lugo
}

Estudio financiado con las ayudas PI06/90432 del Fondo de Investigaciones Sanitarias del Ministerio de Sanidad y PGDIT06BTF91701PR de la Consellería de Industria de la Xunta de Galicia

\section{RESUMEN}

OBJETIVO: El objetivo de este estudio es determinar la repercusión de una consulta monográfica para insuficiencia cardíaca crónica (ICC) y comorbilidad asociada,dirigida por internistas y enfermeria cualificada, sobre la hospitalizacion de estos pacientes.

MATERIAL Y MÉTODOS: Estudio de cohortes prospectivo que incluye 456 pacientes ingresados en la unidad de Medicina Interna con ICC. Al alta los pacientes fueron distribuidos en dos grupos homogéneos, uno control que realizó seguimiento habitual, y otro de intervención que fue seguido en consulta monográfica de ICC. Se realizó un seguimiento desde en-06 hasta abril-09 y se compara en ambas cohortes el número de visitas que realizan a urgencias, reingresos hospitalarios, promedio de reingresos y mortalidad en los ingresos.

RESULTADOS: Los pacientes del grupo intervenido frente a los del grupo control, acudieron a Urgencias significativamente menos (66\% vs $155 \%)(p<0,001)$, reingresaron en menos ocasiones ( $81 \%$ vs $50,5 \%$ ) $(p=0,002)$, el promedio en días de estancia hospitalaria fue significativamente menor (18 días vs 25 días) $(p<0,001)$. Y la mortalidad en los reingresos fue menor $13 \%$ vs $27,8 \%(p<0$ '006).

CONCLUSIONES: El seguimiento por internistas y enfermeria de los pacientes con ICC y comorbilidad, basado en una atención continuada e integral, en la información y en la disponibilidad; es un buen método para disminuir las visitas a urgencias y los reingresos, además de reducir la estancia y mortalidad hospitalaria.

\section{Introducción}

La insuficiencia cardiaca crónica (ICC) en pacientes mayores, es una de las primeras causas de ingreso hospitalario por patología médica en Galicia. Esta alta tasa de hospitalización y el gran gasto asociado, es consecuencia del incremento de los reingresos ${ }^{1}$. El perfil de paciente que reingresa es habitualmente el de un paciente mas anciano, frecuentemente diabético y con importante comorbilidad asociada2,3. Por tanto es un paciente de difícil manejo, que ha sido excluido de los grandes ensayos y como consecuencia, sin evidencias sobre el mejor tratamiento ${ }^{4}$. Sin embargo se conoce que la inclusión de estos pacientes en programas de salud especiales (recomendación I, grado de evidencia A) redunda en una mejora de su calidad de vida ${ }^{5}$.

\section{Abstract}

OBJETIVE: The target of this study is to determine the impact of a monographic consultation for Chronic Heart Failure (CHF) which internists and specialized nursing have a main role, on the hospitalization of those patients. MATERIAL AND METHODS: Prospective cohort study included 456 patients admitted to the Internal Medicine Unit with CHF. Upon discharge patients were divided into two similar groups, one control group that went through routine monitoring, and one intervention group made of patients subject to monitoring through monographic consultation for CHF. The study was conducted from January 6th to April 9th. Both cohorts were compared in terms of the number of urgent service visits, hospital readmissions, readmission rates and mortality average at hospital admission. RESULTS: The number of ER visits of patients subject to monitoring through monographic consultation as opposed to control group was considerably lower ( $66 \%$ vs $155 \%)(p<0,001)$; the readmission rate was significantly reduced ( $81 \%$ vs $50.5 \%)(p=0,002)$; the average hospital stay was significantly lower (18 days vs 25 days) $(p<0,001)$. And the mortality upon readmission was $13 \%$ lower as opposed to $27.8 \%$ ( $p<0$ '006).

CONCLUSION: In CHF and comorbility patients, following a health program in which internists and specialized nursing have a main role and which is based on a continuous and integral tracking, information / training and availability, is a good way to reducing urgents visits and readmissions, as well as reducing hospital stay and mortality.

KEYWORDS: chronic heart failure, comorbidity, monographic consultation.

El objetivo del presente estudio es analizar la posible repercusión sobre la hospitalización, de una consulta monográfica dirigida por internistas y enfermería especializada, para pacientes ancianos con ICC y comorbilidad asociada.

\section{Método}

Pacientes. Estudio prospectivo de cohortes de la totalidad de pacientes ingresados por un episodio de ICC en el servicio de Medicina Interna del Hospital Lucus Augusti de Lugo entre Enero de 2006 y Abril 2009. En el momento de su alta se distribuyeron en dos grupos, grupo de intervención y grupo control. El grupo de intervención se constituyó con los pacientes de 11 Centros de Salud del Área (asignados a un programa de seguimiento en la Consulta Monográfica de 
ICC ). El grupo control se constituyó con los pacientes de los restantes Centros de Salud.

Seguimiento y recogida de datos. En el grupo de intervención los pacientes se citaron en una consulta monográfica de ICC. La labor en consulta es realizar un seguimiento integral, cubriendo la mayoría de los problemas del paciente; y continuado, con consultas al principio frecuentes que permitan la implementación terapéutica y consultas no programadas para atender situaciones de descompensación. Además se realiza la formación e información del paciente para lograr mejores niveles de adherencia terapéutica y de auto-control. En el grupo control no se realizó ningún tipo de intervención diferente a la habitual.

Para ambos grupos se contabilizaron el número de visitas a urgencias, las fechas de ingreso y alta de los reingresos hospitalarios y la mortalidad. Para ello se consultaron los registros informáticos del Centro.

Análisis estadístico: La totalidad de los datos se incluyeron en una base de datos diseñada al efecto. En el análisis descriptivo utilizamos las técnicas habituales. En la comparación de dos variables cuantitativas utilizamos el test T de Student, previa evaluación de la homocedasticidad. En la comparación múltiple de medias se utilizó ANOVA aunque en los casos de distribución no normal se utilizó la prueba de Kruskal-Wallis. Para la totalidad del análisis utilizamos el paquete estadístico SPSS ${ }^{6}$. El nivel de significación estadística se estableció en $p<0,05$

\section{Resultados}

Incluimos un total de 456 pacientes, con predomino de mujeres (51\%) y siendo la edad media 78,8 (DS 10,3) años. De ellos 93 fueron seguidos en la consulta monográfica de ICC y 363 en circuitos habituales. Las características generales de ambos grupos se recogen en la Tabla 1.

Tabla 1. Características y comparación de las cohortes estudiadas

\begin{tabular}{|l|c|c|c|}
\hline & $\begin{array}{c}\text { Grupo control } \\
(\mathrm{n}=363)\end{array}$ & $\begin{array}{c}\text { Grupo } \\
\text { intervención } \\
(\mathrm{n}=93)\end{array}$ & $p$ \\
\hline Sexo (\% varones) & $51,2 \%$ & $51 \%$ & n.s. \\
\hline Edad (media DT) & $78,611,2$ & 799,4 & n.s. \\
\hline HTA & $247(68 \%)$ & $64(68,8 \%)$ & n.s. \\
\hline DM-II & $119(32,8 \%)$ & $27(29 \%)$ & n.s. \\
\hline EPOC & $147(40,5 \%)$ & $33(33,5 \%)$ & n.s. \\
\hline Fibrilación auricular & $131(36,1 \%)$ & $36(38,7 \%)$ & n.s. \\
\hline Cardiopatía isquémica & $43(11,8 \%)$ & $11(11,8 \%)$ & n.s. \\
\hline Cardiopatía hipertensiva & $93(25,6 \%)$ & $25(26,9 \%)$ & n.s. \\
\hline FE preservada & $273(75,2 \%)$ & $69(74,2 \%)$ & n.s. \\
\hline 3 ó más patologías & $201(55,4 \%)$ & $47(59,3 \%)$ & n.s. \\
\hline 5 ó más patologías & $161(44,3 \%)$ & $38(40,9 \%)$ & n.s. \\
\hline
\end{tabular}

DT: desviación típica. FE: fracción de eyección. ns no significativo
Con respecto a los resultados asistenciales, los pacientes del grupo de intervención acudieron menos veces a urgencias $(p<0,001)$, reingresaron menos veces $(p=0,002) y$, cuando lo hicieron, sus estancias fueron significativamente menores $(p<0,001)$ (Tabla2). El riesgo relativo de reingreso asociado al seguimiento en la consulta de ICC se redujo a 0'22 (IC 95\% 0'04-1.00). Ésto es, el número de consultas necesarias para evitar un reingreso es de 4'2. Con respecto a la reducción del tiempo de estancia hospitalaria, se reduce en un $26 \%$ del tiempo de ingreso.

Por último, la mortalidad en el grupo de intervención fue inferior a la observada en el grupo control (13\% vs. $27,8 \%)$ $(p<0$ '006).

Tabla 2. Comparación entre el grupo de control y el grupo de intervención para las siguientes variables: número de veces que acuden a urgencias, número de reingresos, promedio de días de ingreso y mortalidad en el ingreso

\begin{tabular}{|l|c|c|c|}
\hline & $\begin{array}{c}\text { Grupo control } \\
(n=299)\end{array}$ & $\begin{array}{c}\text { Grupo } \\
\text { intervención } \\
(n=69)\end{array}$ & $p$ \\
\hline Urgencias & $764(155 \%)$ & $46(66,6 \%)$ & $p<0.001$ \\
\hline Reingresos & $245(81 \%)$ & $44(63 \%)$ & $p<0.002$ \\
\hline $\begin{array}{l}\text { Dias de ingreso } \\
\text { (media DT) }\end{array}$ & 2522.9 & 1812.8 & $p<0.001$ \\
\hline Mortalidad & $27,8 \%$ & $13 \%$ & $p<0,006$ \\
\hline \multicolumn{4}{|c|}{ DT: desviación típica } \\
\hline
\end{tabular}

\section{Discusión}

Este estudio muestra que los pacientes más mayores con ICC y comorbilidad asociada, manejados en una consulta monográfica de Medicina Interna, acuden menos a urgencias, ingresan menos y, cuando lo hacen, tienen menor estancia y mortalidad que los pacientes atendidos por el modelo tradicional.

A diferencia del sistema tradicional, en la consulta monográfica se realiza un seguimiento continuado, adaptado a las necesidades del paciente; los internistas ofrecen una atención integral al manejar la mayoría de las comorbilidades asociadas; la enfermería aporta información y formación al paciente y/o cuidadores, mejorando el conocimiento de su enfermedad, la adherencia terapéutica y la detección precoz de los síntomas de alarma ${ }^{7}$. También ofrece accesibilidad mediante comunicación telefónica que permite corregir a tiempo procesos de descompensación y evitar situaciones más extremas.

Los pacientes atendidos por Medicina Interna son pacientes diferentes a los manejados por cardiología, presentan cardiopatías no complejas de manejo (no requieren técnicas invasivas), pero son clínicamente mas difíciles, al ser mas ancianos, tolerar peor los fármacos, ser frecuentemente dia- 
béticos y acompañarse de otras comorbilidades ${ }^{8}$. Por todo ello, el manejo integral de la pluripatologia y un seguimiento más continuado es lo más aconsejable. En nuestro estudio de cohortes, ambos grupos están formados por pacientes que obedecen a este perfil y al compararlos no existen diferencias significativas (tabla 1).

Nuestros resultados objetivan que la implantación de este programa, y la optimización terapéutica según guía clínica ${ }^{9}$, juega un papel en la evolución de la enfermedad ${ }^{10}$.

La situación de ancianidad, comorbilidad e ICC hace imposible evitar la totalidad de los reingresos hospitalarios ${ }^{11}$ a lo largo de los pocos años de vida que les restan a estos pacientes ${ }^{12,13}$. Sin embargo, la mejora de la calidad de vida que logra la consulta monográfica, se traduce en una reducción de las visitas a urgencias y de reingresos hospitalarios (Tabla 3).

Probablemente este modelo de asistencia genera un mayor número de visitas a las consultas, tanto programadas como de demanda urgente; pero permite un mejor control de su ICC, y posibilita una atención mas temprana en las fases de descompensación. Todo ello permite reducir ingresos hospitalarios. De hecho por cada cuatro consultas realizadas se evita un ingreso (NNT 4,2).

$Y$ no solamente reingresan menos, sino que el promedio de días de ingreso hospitalario es significativamente menor en los pacientes adheridos al seguimiento.

Además una gran parte de los pacientes del grupo intervención ingresan en nuestro servicio, donde conocemos al paciente y nos da ventaja a la hora del manejo hospitalario, evitando iatrogenias y complicaciones. Tanto es así que demostramos disminución no solo de la estancia, sino también de la mortalidad en los reingresos hospitalarios.

En definitiva un sistema de seguimiento adaptable a la necesidad del paciente, con una visión integral, una consulta de enfermería implicada entre otras en la información y control del paciente, y por ultimo el autocontrol del propio paciente; es la base para mantener la estabilidad del paciente, disminuir los reingresos hospitalarios y en definitiva mejorar la calidad de vida

Por otra parte, los recursos empleados no suponen una inversión excesiva por parte del sistema. Implantar un programa de este tipo supone una diferente organización del personal integrado en el servicio y un cambio en el enfoque del pacien- te con ICC. Además en términos de rentabilidad económica la disminución de los ingresos supone un ahorro de recursos hospitalarios que son costosos y en ocasiones, escasos.

Puesto que el trabajo consiste en optimizar las medidas ya conocidas (tratamientos y sistemas de control), sus principales ventajas son: es muy barata, y se integra bien, fácil y rápidamente en el trabajo habitual.

No se incluyeron análisis coste-efectividad, sin embargo, la reducción significativa de los reingresos, y en la estancia global hace pensar que la relación coste-eficacia se inclina a favor del grupo intervención ${ }^{14}$.

Por todo ello, y para la población descrita, una consulta monográfica de ICC integrada en una sección de medicina interna y funcionando con internistas, con apoyo de enfermería cualificada, es mejor que el sistema tradicional, ya que consigue reducir las visitas a urgencias, reingresos y estancias hospitalarias.

\section{Bibliografia}

1. Rodríguez F, Guallar P, Banegas JR, Del Rey J. Trends in hospitalization and mortality for heart failure in Spain, 1980-1993. Eur Heart J. (1997).18:1771-9.

2. Montes J, Lado M, Rey G, et al. Demografía de la insuficiencia cardiaca en Galicia. Análisis de diferentes poblaciones y ámbitos asistenciales Galicia Clin 2008; 69 (1): 7-10.

3. Conthe P, Montoto C, Forteza J, et al. Comisión redactora SEMI-IC. Grupo de trabajo de insuficiencia cardiaca de la Sociedad Española de Medicina interna (SEMI). La Insuficiencia Cardiaca en los servicios de medicina Interna (estudio SEMI-IC). Med Clin (Barc) 2002 ; 118: 605-10

4. Rich M W. Office Management of heart failure in the elderly. Am Journal Med 2005 (118:342-348).

5. Dickstein K, Cohen-Solal A, Filippatos G, et al. ESC guideliness for the diagnosis and treatment of acute and chronic heart failure 2008. Eur Heart J (2008).29: 2388 2442.

6. SPSS inc. SPSS for windows ver 15.0.Chicago IL: SPSS, 1999.

7. Abeledo MC. Mosquera E. Cerqueiro J. et al. La consulta de enfermería en el control de pacientes con insuficiencia cardíaca. $2^{\circ}$ Congreso nacional de Atención Sanitaria al Paciente Crónico. Santiago de Compostela. 2010

8. Montero $\mathrm{M}$ et al. Comorbilidad de los pacientes ingresados por insuficiencia cardíaca en los servicios de Medicina Interna. Rev Clín Esp. 2010;210:149-58.

9. Mariell MD, William T. Abraham MD, et al. 2009 Focused Update: ACCF/AHA Guidelines for the Practice Guideline: Focused Update. Diagnosis and Management of Heart Failure in Adults. A Report of the American College of Cardiology Foundation/ American Heart Association Task Force on Practice Guidelines. 2009. Circulation 2009;119;1977-2016.

10. Marc A, Pfeiffer MD, Kart MD, et al. Effects of candesartan on mortality and morbidity in patients with chronic heart failure: the CHARM-Overall programme. The Lancet 2003, $759-766$.

11. Krumholz HM, Chen Y, Wang Y, et al. Predictors of readmissions among elderly survivors of admission with heart failure. Am Heart J. 2000;139:72-7.

12. Owen Andrew FRCP et al. Life expectancy of elderly and very elderly patients with chronic heart failure. Am Heart J. 2006.151(6):1322e1-1322e4.

13. Goldberg R, Ciampa J, Lessard D,et al. Long-term Survival After Heart Failure: A Contemporary Population-Based Perspective. Arch Intern Med. 2007; 167:490-496.

14. Anguita M. Programas de intervención en la insuficiencia cardiaca: análisis crítico. Rev Esp Cardiol 2007; 7: 45 - 56 